Supporting Information

\title{
Controlled Nanoscale Cracking of Graphene Ribbons by Polymer Shrinkage
}

Anjishnu Sarkar, ${ }^{\dagger}$ Debadrita Paria, ${ }^{\star}$ Ishan Barman, ${ }^{\ddagger},{ }^{\dagger}, \nabla$ David H. Gracias ${ }^{\dagger,}, \|, \S *$

†Department of Chemical and Biomolecular Engineering, Johns Hopkins University. 3400 North Charles Street, Baltimore, MD 21218, United States.

Department of Mechanical Engineering, Johns Hopkins University. 3400 North Charles Street, Baltimore, MD 21218, United States.

"Department of Materials Science and Engineering, Johns Hopkins University. 3400 North Charles Street, Baltimore, MD 21218, United States.

${ }^{\S}$ Department of Chemistry, Johns Hopkins University. 3400 North Charles Street, Baltimore, MD 21218, United States.

${ }^{\dagger}$ Department of Oncology and ${ }^{\nabla}$ The Russell H. Morgan Department of Radiology and Radiological Science, The Johns Hopkins University School of Medicine, Baltimore, Maryland 21287, United States

*Corresponding author: dgracias@jhu.edu 


\section{Fabrication and patterning schematics}
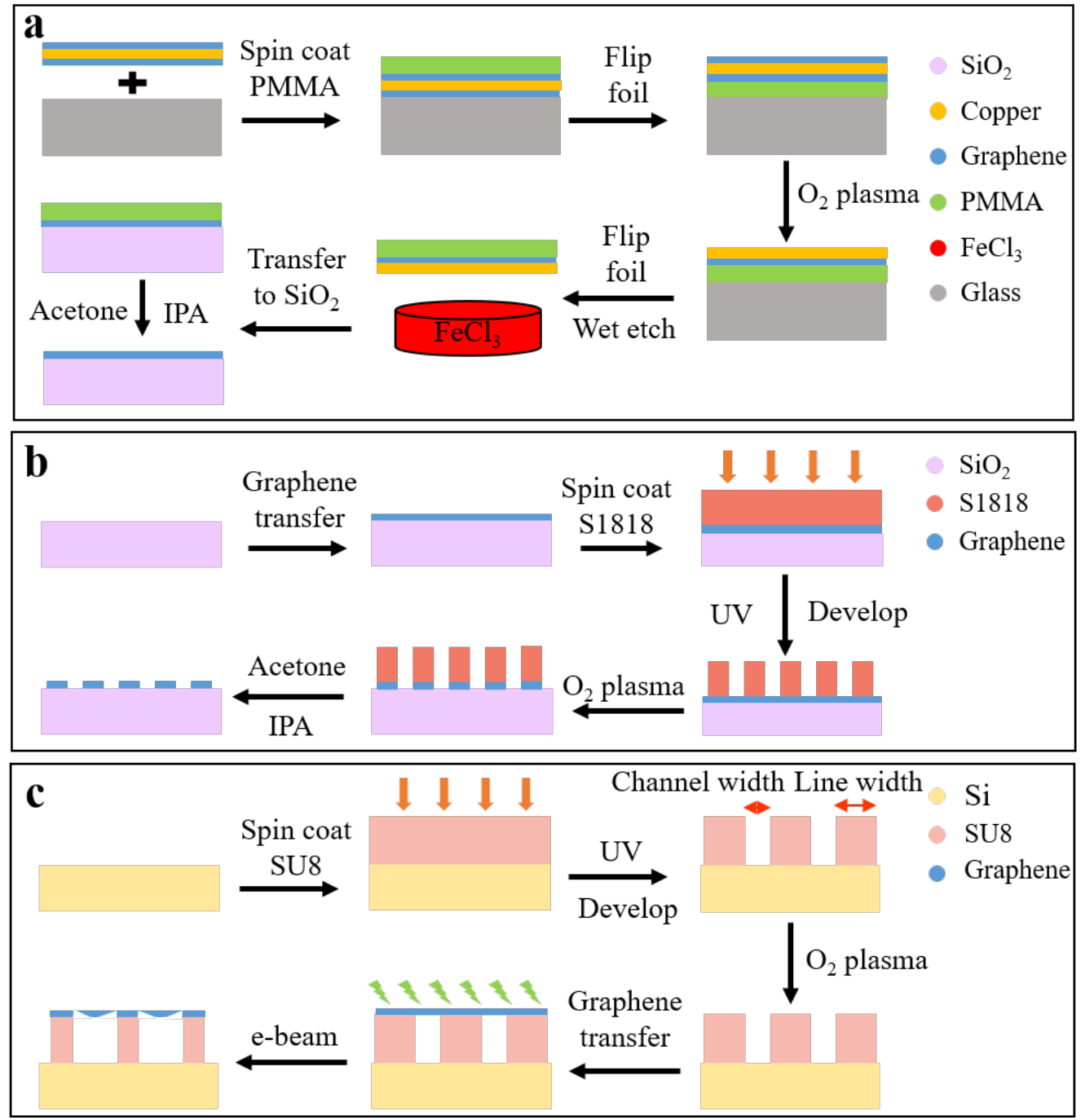

Figure S1. Schematics of, (a) PMMA assisted wet transfer of CVD graphene from copper to the $\mathrm{SiO}_{2}$ surface. (b) Micropatterning of graphene on a $\mathrm{SiO}_{2}$ substrate. (c) Transfer of patterned graphene microribbons on SU8 lines followed by e-beam mediated shrinkage of SU8 lines, expansion of SU8 channel width and breakage of graphene microribbons. 


\section{Crack-free, suspended graphene microribbons after transfer on micropatterned SU8}

\section{lines}

We patterned $3 \mu \mathrm{m}$ wide graphene ribbons and subsequently transferred them on SU8 lines in an oblique fashion (Figure S2(a)). We used scanning electron microscopy at a low probe current and at a tilt angle of $30^{\circ}$ to ensure that graphene ribbons did not crack prior to controlled breakage at a higher probe current (Figure S2(b)). The Raman spectra of the suspended graphene ribbons show the signature of crack-free graphene and the defect peak (D) at $1350 \mathrm{~cm}^{-1}$ is absent (Figure S2(c)).
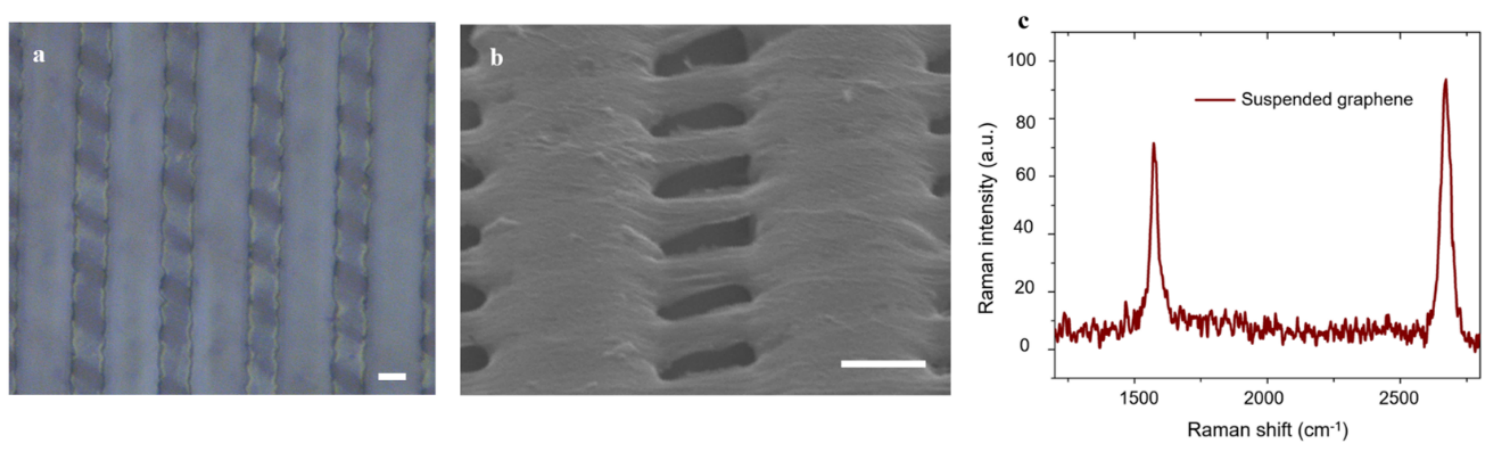

Figure S2. Micrographs of micropatterned graphene ribbons before cracking (a) Bright field optical micrograph of $3 \mu \mathrm{m}$ wide graphene ribbons suspended on $8 \mu \mathrm{m}$ wide SU8 lines (b) Isometric SEM micrographs of crack-free, suspended ribbons taken at an angle of $30^{\circ}$. The scale bar is $4 \mu \mathrm{m}$. (c) Raman spectra of crack-free, suspended graphene microribbons on SU8. 


\section{Determination of the best permutation for achieving highest SU8 channel width}

The rise in the spacing between two adjacent SU8 lines (channel width) is a latent parameter that is directly proportional to the extent of breakage in graphene. The SU8 channel width is governed by three factors, namely UV crosslinking intensity, temperature, and e-beam dose. To better understand the effect of each of the aforementioned factors on SU8 channel width, we carried out $3^{3}=27$ experiments, which correspond to 27 unique permutations. Each experiment was conducted in triplicates, and we report the average value of SU8 channel width (listed as Table S1). We observe that the channel width attains its minimum value for a UV crosslinking intensity of 125 $\mathrm{mJ} / \mathrm{cm}^{2}$ and a low e- beam dose (experiments 19 and 25). The SU8 channel width reaches its highest value for UV crosslinking intensity of $50 \mathrm{~mJ} / \mathrm{cm}^{2}$ and a high e-beam dose (experiment 6). This shows that SU8 channel width increases with e-beam dose and decreases with UV crosslinking. 
Table S1. Evolution of SU8 channel width for 27 permutations of UV crosslinking intensity, Temperature and e-beam dose.

\begin{tabular}{|c|c|c|c|c|}
\hline Experiment & $\begin{array}{l}\text { UV crosslinking intensity } \\
\qquad\left(\mathrm{mJ} / \mathrm{cm}^{2}\right)\end{array}$ & $\begin{array}{c}\text { Temperature } \\
\left({ }^{\circ} \mathrm{C}\right)\end{array}$ & $\begin{array}{l}\text { e-beam dose } \\
\text { (a.u.) }\end{array}$ & $\begin{array}{l}\text { SU8 channel width } \\
(\mu \mathrm{m})\end{array}$ \\
\hline 1 & 50 & No heating & Low & 6.7 \\
\hline 2 & 50 & No heating & Med & 6.8 \\
\hline 3 & 50 & No heating & High & 7.8 \\
\hline 4 & 50 & 90 & Low & 8.7 \\
\hline 5 & 50 & 90 & Med & 9.0 \\
\hline 6 & 50 & 90 & High & 9.5 \\
\hline 7 & 50 & 200 & Low & 7.7 \\
\hline 8 & 50 & 200 & Med & 8.1 \\
\hline 9 & 50 & 200 & High & 8.5 \\
\hline 10 & 85 & No heating & Low & 7.3 \\
\hline 11 & 85 & No heating & Med & 7.9 \\
\hline 12 & 85 & No heating & High & 8.3 \\
\hline 13 & 85 & 90 & Low & 7.7 \\
\hline 14 & 85 & 90 & Med & 8.3 \\
\hline 15 & 85 & 90 & High & 8.8 \\
\hline 16 & 85 & 200 & Low & 8.0 \\
\hline 17 & 85 & 200 & Med & 8.1 \\
\hline 18 & 85 & 200 & High & 8.3 \\
\hline 19 & 125 & No heating & Low & 5.2 \\
\hline 20 & 125 & No heating & Med & 5.6 \\
\hline 21 & 125 & No heating & High & 5.9 \\
\hline 22 & 125 & 90 & Low & 5.5 \\
\hline 23 & 125 & 90 & Med & 6.1 \\
\hline 24 & 125 & 90 & High & 6.7 \\
\hline 25 & 125 & 200 & Low & 5.3 \\
\hline 26 & 125 & 200 & Med & 5.7 \\
\hline 27 & 125 & 200 & High & 6.0 \\
\hline
\end{tabular}




\section{Determination of the absorption current corresponding to the e-beam doses "low", "med" and "high" using a Faraday cup}

We measured the approximate values of the three distinct e-beam doses as follows. All our e-beam experiments on e-beam induced SU8 shrinkage were conducted using a tungsten filament thermionic emission JEOL JSM IT100 SEM. Since it was not readily possible to install a Faraday cup on this SEM, we also reproduced the SU8 shrinkage experiments using a Thermo Scientific Helios G4 UC Focused Ion Dual Beam instrument which consists of an e-beam for SEM. We installed a Faraday cup to the latter. To ensure reproducibility, we irradiated SU8 lines UV crosslinked with $80 \mathrm{~mJ} / \mathrm{cm}^{2}$ with the e-beam at different probe currents, as measured by the Faraday cup (Figure S3). We maintained the identical working distance $(11 \mathrm{~mm})$, magnification $(6000 \mathrm{X})$ and acceleration voltage $(20 \mathrm{kV})$ as the original experiments. For each probe current, we increased the duration of exposure to match the rise in SU8 channel width as obtained with each of "low", "med", and "high" e-beam doses. Listed below are the charge per unit area values of the e-beam doses.

Low e-beam dose: $\quad$ Charge per unit area $<0.5 \mathrm{mC} / \mathrm{cm}^{2}$

Med e-beam dose: Charge per unit area $\sim 366.1 \mathrm{mC} / \mathrm{cm}^{2}$

High e-beam dose: Charge per unit area $>915.1 \mathrm{mC} / \mathrm{cm}^{2}$ 
a

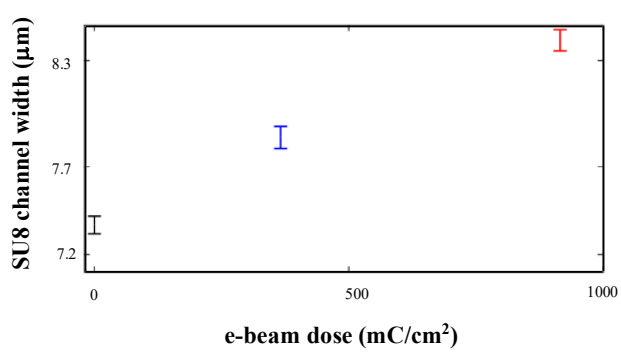

b

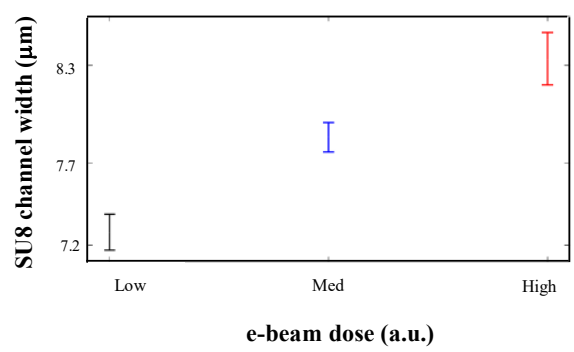

Figure S3. Identification of the e-beam doses which results in identical increase in SU8 channel width for resist photocrosslinked with $85 \mathrm{~mJ} / \mathbf{c m}^{2}$. (a) Variation of SU8 channel width with e-beam dose measured with a Faraday cup in $\mathrm{mC} / \mathrm{cm}^{2}$. (b) Variation of SU8 channel width at different e-beam dose 


\section{Effect of e-beam dose on $5 \mu \mathrm{m}$ wide graphene microribbons}

We repeated the fabrication corresponding to Figure 2, and irradiated the samples at the three ebeam doses (Figure S4 (d-f)). We observed the formation of a narrow slit for the med e-beam dose, followed by a widening of the slit at the high e-beam dose.
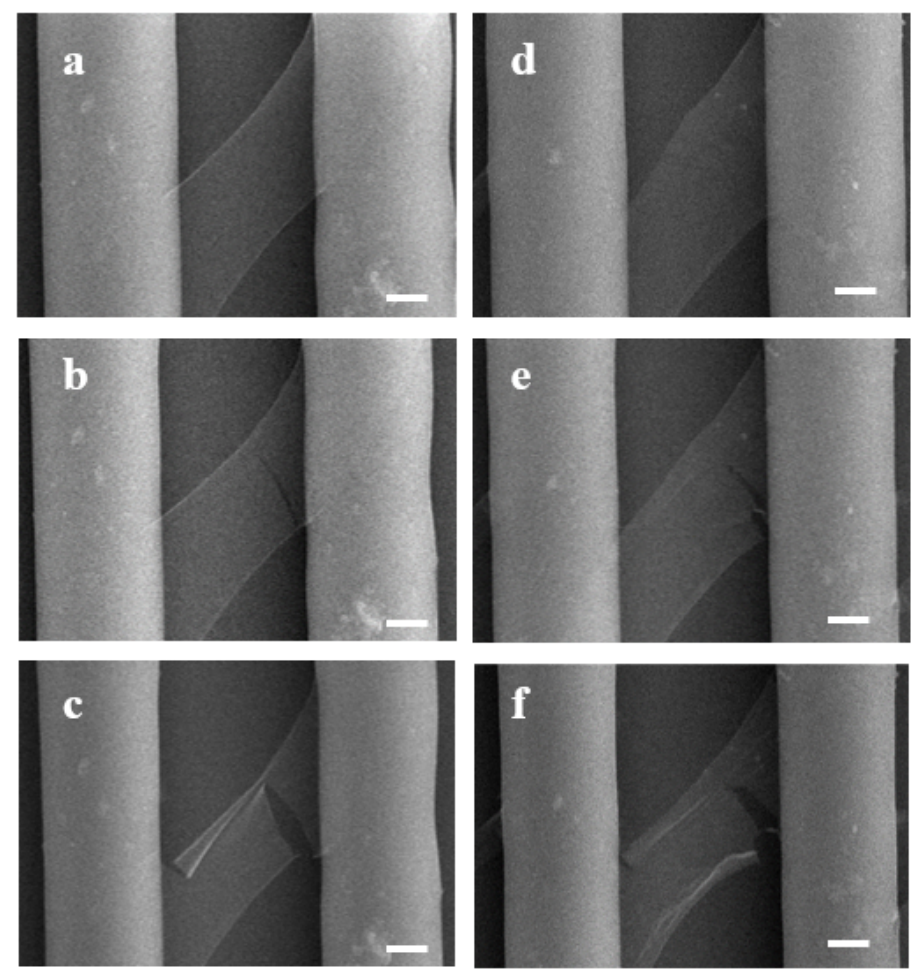

Figure S4. Evolution of rise in SU8 channel width and consequent breakage of graphene microribbons. SEM micrographs showing progressive stages of graphene breakage for microribbon 1 (ac) and microribbons $2(\mathrm{~d}-\mathrm{f})$. The scale bar is $2 \mu \mathrm{m}$. 


\section{Control experiments: Expansion of the SU8 channel width in the absence of graphene}

We patterned SU8 channels with a channel width of $6.4 \mu \mathrm{m}$ (Figure S5 (a)) and subjected them to e-beam irradiation. At medium e-beam dose, the channel width increases to $7 \mu \mathrm{m}$ and remains constant at high e-beam dose.
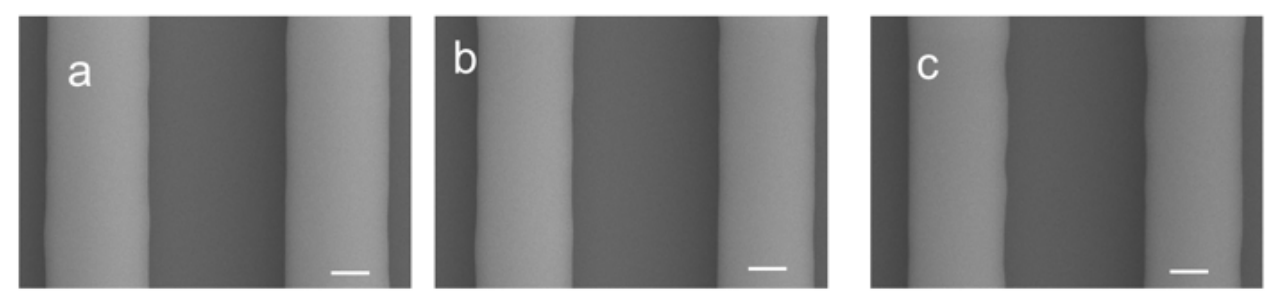

Figure S5. SEM micrographs of a SU8 channel before and after irradiation with the e-beam (a) SU8 at low e-beam dose (b) medium e-beam dose. (c) high e-beam dose. The scale bar is $2 \mu \mathrm{m}$. 


\section{Nanoscale cracks developed in $8 \mu \mathrm{m}$ wide, suspended graphene microribbons}

We patterned $8 \mu \mathrm{m}$ wide graphene ribbons with $5 \mu \mathrm{m}$ spacing and subsequently transferred them on SU8 lines in an oblique fashion (Figure S6 (a)). At medium e-beam dose, a narrow slit forms (Figure S6 (b)), which assumes a length of $400 \mathrm{~nm}$ upon irradiation with the high e-beam dose (Figures S6 (c) and (d)).
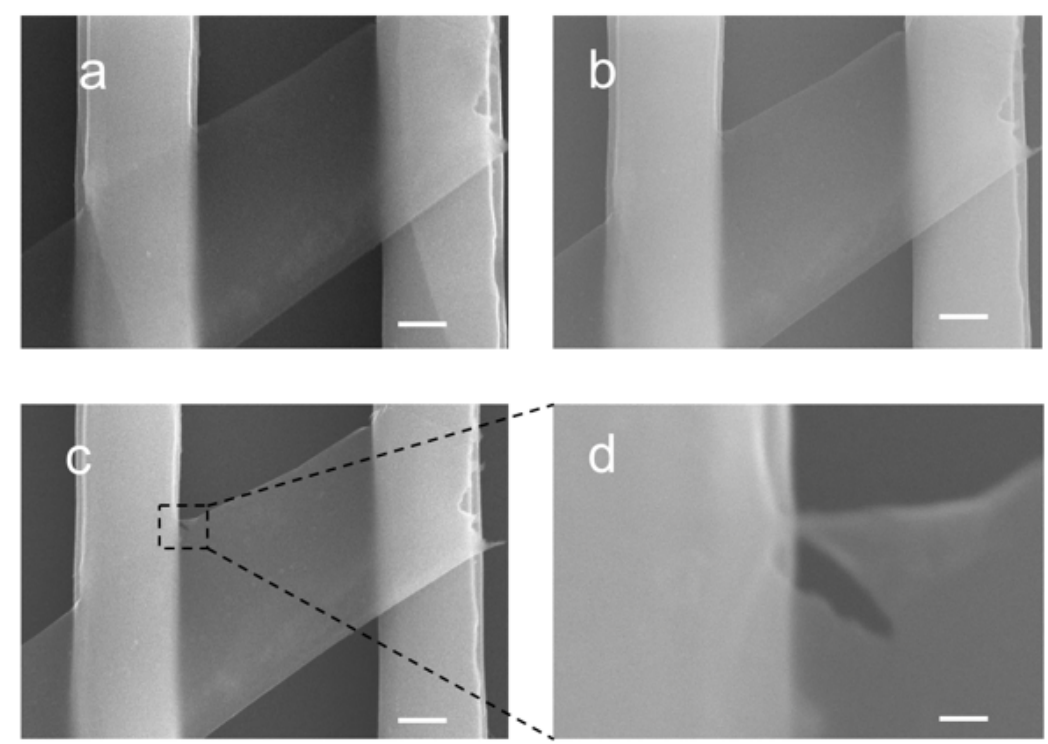

Figure S6. SEM micrographs of $8 \mu \mathrm{m}$ wide graphene microribbons before and after cracking (a) SEM showing supported and suspended regions (b) SEM of suspended graphene at medium e-beam dose. (c) $200 \mathrm{~nm}$ crack at the intersection of suspended graphene and SU8 at high e-beam dose. (d) Zoomed in image of graphene and SU8. The scale bar is $2 \mu \mathrm{m}$ for a-c and $200 \mathrm{~nm}$ for $\mathrm{d}$. 


\section{Note on the spread in the standard deviation of the response parameter}

The spread in the standard deviations for Figure 3a and Figure 4a can be attributed to two reasons. First, to discretize the effect of each factor for one of three levels, we choose the permutations which correspond to a distinct level for a given factor. Hence, the mean value for a factor at a level is governed by several permutations of the other two factors across different levels, which causes the standard deviation to be high. Secondly, as shown in Figure 2a, upon increasing the e-beam dose from medium to high, the length of the nanoscale crack remains constant while the area of the cracks increases by a factor of seven. Since the percentage of graphene cracking is measured as the ratio of the cracked slit length to the original ribbon width $(5 \mu \mathrm{m})$, the increase in area is not accounted for in the percentage cracking. This causes the standard deviation to assume negative percentages in Figure 4a. However, we calculate the signal-to-noise $(\mathrm{S} / \mathrm{N})$ ratios for the response parameters across the levels to gain a better insight of the robustness of the DOE. ${ }^{1} \mathrm{We}$ show in Figures 5 and 6 that the UV crosslinking plays a dominant role in the widening of SU8 channels, and the consequent breakage of graphene microribbons. 


\section{Note on Video S1}

Video S1 is a video showing the in situ, complete breakage of a suspended graphene microribbon in real time, taken in an SEM. The SU8 lines were photopatterned with a UV crosslinking intensity of $125 \mathrm{~mJ} / \mathrm{cm}^{2}$ and annealed on a hotplate at $90{ }^{\circ} \mathrm{C}$ prior to the transfer of the graphene microribbon. The SU8 lines have a width of $7.6 \pm 0.1 \mu \mathrm{m}$, and a channel width of $5.8 \pm 0.0 \mu \mathrm{m}$. We observe that a crack appears in the graphene microribbon in approximately 45 seconds (which corresponds to an e-beam dose between $0.5 \mathrm{mC} / \mathrm{cm}^{2}$ and $27.4 \mathrm{mC} / \mathrm{cm}^{2}$ ), and the graphene microribbon cracks completely in approximately 58 seconds (which corresponds to an e-beam dose between 27.4 $\mathrm{mC} / \mathrm{cm}^{2}$ and $\left.35.3 \mathrm{mC} / \mathrm{cm}^{2}\right)$. 


\section{REFERENCES}

1. Mahapatra, S. S.; Chaturvedi, V., Modelling and analysis of abrasive wear performance of composites using Taguchi approach, Int. J. Eng. Sci. Technol., 2009, 1(1) 123-135. 